Blicharz Agnieszka, Marzeda Magdalena, Drozd Małgorzata, Czarnota Jakub, Piecewicz-Szczesna Halina. Beriberi disease - a picture of thiamine deficiency in underdeveloped and highly developed countries. Journal of Education, Health and Sport. 2020;10(9):407-414. eISSN 2391-8306. DOI http://dx.doi.org/10.12775/JEHS.2020.10.09.048

https://apcz.umk.pl/czasopisma/index.php/JEHS/article/view/JEHS.2020.10.09.048

https://zenodo.org/record/4035767

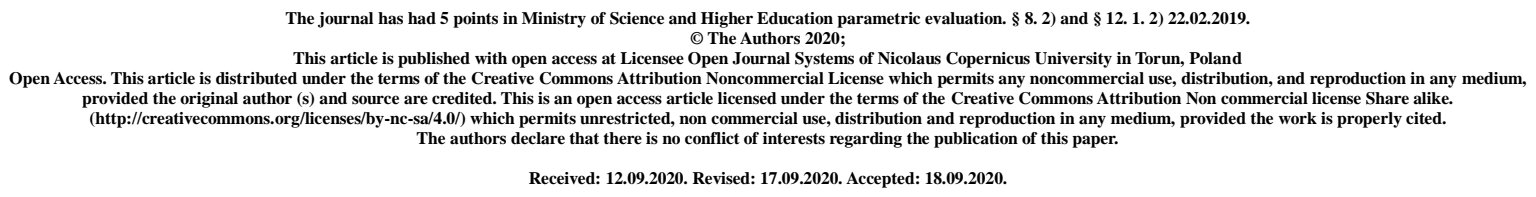

\title{
Beriberi disease - a picture of thiamine deficiency in underdeveloped and highly developed countries
}

\section{Agnieszka Blicharz ${ }^{1}$, Magdalena Marzęda ${ }^{1}$, Małgorzata Drozd ${ }^{1}$, Jakub Czarnota ${ }^{1}$, Halina Piecewicz-Szczęsna ${ }^{2}$}

${ }^{1}$ Student Research Circle at the Department of Epidemiology and Clinical Research Methodology, Medical University of Lublin

${ }^{2}$ Department of Epidemiology and Clinical Research Methodology, Medical University of Lublin

Corresponding author: Agnieszka Blicharz, e-mail: agnieszkablicharz9603@gmail.com

\section{ORCID ID:}

Agnieszka Blicharz https://orcid.org/0000-0003-4536-0651; agnieszkablicharz9603@gmail.com

Magdalena Marzęda https://orcid.org/0000-0003-4397-5214; mmarzeda@ gmail.com Małgorzata Drozd https://orcid.org/0000-0002-0710-2451; drozd.malg@ gmail.com Jakub Czarnota https://orcid.org/0000-0003-2783-0349; kubbaa232@interia.pl Halina Piecewicz-Szczęsna https://orcid.org/0000-0002-0573-7226; halpiec@wp.pl 


\begin{abstract}
:
Introduction and purpose: Thiamin (Vitamin B1) is a water-soluble vitamin. It plays an important role in many biological processes, mainly in glucose metabolism. Its deficiency is called beriberi disease. Beriberi was discovered at the turn of the 19th and 20th centuries and is now a disease that occurs in both underdeveloped and highly developed countries.

Brief description of the state of knowledge: Thiamine deficiency can affect the cardiovascular, nervous and immune systems, which is seen as dry, wet, fulminant beriberi or as Wernicke-Korsakoff syndrome. Worldwide, vitamin B1 deficiency is most commonly reported in populations where rice and ground grains are the main food source, but also in patients with chronic alcohol abuse, diabetes, gastrointestinal surgery, cancer, and patients undergoing chronic diuretic therapy, which increases urine loss.

Summary: Treatment of beriberi disease consists in supplementing vitamin B1 in appropriate doses. The symptoms of thiamine deficiency are non-specific, however, early diagnosis and initiation of vitamin administration quickly lead to clinical improvement. It should be remembered that thiamine deficiency is often not included in the differential diagnosis of complaints reported by patients. This may be due to the belief that diets in European countries containing food products enriched with vitamins exclude clinically evident micronutrient deficiencies.
\end{abstract}

Key words: beriberi; thiamin deficiency; Korsakoff syndrome; Wernicke encephalopathy

\title{
Introduction and purpose
}

Beriberi disease is caused by a deficiency of vitamin B1 - thiamine. Thiamin plays an important role in the transport of electrolytes, in the synthesis of neurotransmitters, and in glucose metabolism. Thiamin must be supplied with food on a daily basis because the body does not store much of it. People on a diet low in thiamin or with malabsorption can exhaust their stores in just 2-3 weeks [1]. Vitamin B1 deficiency can cause functional and morphological changes in the nervous system. With long-term thiamine deficiency in the body, beriberi can develop. This disease mainly affects people in Asian countries on a monotonous, low-vitamin diet, often consuming, for example, white rice [2]. Beriberi is also present in European countries. It occurs especially in malnourished people, with alcoholism, with refeeding syndrome, after gastrointestinal surgery, and in pregnant women, especially during uncontrolled vomiting. The increased risk of thiamine deficiency should also be borne in mind in patients with severe burns, extensive surgery, septic shock, end-stage renal disease and heart failure [3].

Beriberi comes in three forms: dry (chronic), in which symptoms from the nervous system dominate, wet (acute), in which the cardiovascular system is damaged, and lightning (shoshin beriberi), in which disorders such as wet, but with a more severe course [4].

There may also be different forms of the disease:

(1) breaking out in groups; (2) appearing during the course of a febrile illness or puerperium;

(3) complicating cachexia due to neoplasm; (4) a variant characterized by amblyopia, difficulty, and ataxia; (5) infantile beriberi; (6) childhood beriberi [2]. 
There is no specific concentration of thiamine at which symptoms of its deficiency will appear, so it is important to remember about the symptoms of beriberi when diagnosing a patient's ailments. The diagnosis of the dry form of beriberi is based on neurological symptoms. These include: brisk tendon reflexes, peripheral neuropathy, and / or polyneuritis with or without parasthesias, muscle weakness and / or pain of the upper and lower extremities, gait ataxia, and convulsions. Initially, neuropathy usually affects the lower limbs, in more advanced stages, it may also affect the upper limbs. Moist beriberi is manifested by tachycardia or bradycardia, lactic acidosis, dyspnoea, hypertrophy and dilatation of the heart (especially of the right ventricle), respiratory failure, edema of the lower extremities and systemic venous hypertension [5].

The form of Shoshin beriberi (from the Japanese sho meaning acute damage, and shin meaning heart), i.e. a fulminant variant, can occur with severe two-chamber failure, metabolic acidosis, variable cardiac output with vascular collapse, peripheral cyanosis, and eventually leads to death [6].

Wernicke-Korsakoff syndrome is also caused by a deficiency of thiamine in the body, mainly due to alcoholism. However, the symptoms are different from beriberi. It consists of two teams:

- Wernicke encephalopathy- acute, reversible state of confusion with clinical symptoms

- Korsakoff's syndrome- irreversible confabulations, gait disturbances and memory loss

Wernicke-Korsakoff syndrome is thought to have a classic triad of symptoms including ataxia, nystagmus and an altered mental state (confusion / dementia), but this can be seen only in about $1 / 3$ of patients [7].

The aim of the study is to review the data on the history and etiology of beriberi as well as to compare the image and causes of the disease in poor and well-developed countries.

\section{Description of the state of knowledge}

\section{A short history of beriberi}

The turn of the 19th and 20th centuries was the period of many discoveries, also in the field of medicine. European scientists were fascinated by tropical diseases and research on them, so expeditions were organized to set up hospitals in poor parts of the world and to conduct experiments and tests on previously unknown diseases. Among them is the Dutch doctor Christiaan Eijkman, who was interested in beriberi.

At the end of the 19th century, little was known about beriberi. There were characteristic neurological symptoms such as: problems with walking, tingling, loss of sensation, paralysis of the lower limbs, mental disorientation. Moreover, it has been observed that the disease appears focal. Beriberi was a very common disease in the Dutch India where Eijkman came to find out the cause of the disease. He began his research with the assumption that the cause of the disease was bacterial in origin because it was focal. However, after many experiments with chickens, he had to dismiss the idea. The progress in the work on understanding the cause of the disease resulted from the researcher's luck and accident. The chickens, previously fed with white rice, later they were fed rough rice. Their neurological symptoms, similar to those found in people with beriberi, began to subside. Eijkman noticed how changing the diet affected the health of the animals. 
Thanks to this, he changed the direction of research, which resulted in promising results. $\mathrm{He}$ performed an autopsy of sick chickens, on the basis of which he described typical histological changes, he proved that the disease was not caused by changes in blood, respiration, temperature or climate. He concluded that the rice husk component protects against the occurrence of beriberi. For his contribution to the development of medicine, he was awarded the Nobel Prize in 1929. However, he never isolated an ingredient that prevents beriberi from developing. This merit is attributed to Kazimierz Funk, who in 1911 discovered thiamine and included it in a group of compounds necessary for life, which he had invented, called "vitamins".

\section{The picture of beriberi disease in underdeveloped countries}

At the turn of the 20th century, the process of grinding rice was considered a great discovery and gift from Europeans to the people of Asian countries. However, in populations dominated by low-varied and monotonous diets, this initiative had unexpectedly bad results. In Asia, the diet is based on rice, the shell of which contains thiamine. Husked rice is devoid of this vitamin. Beriberi develops when there is no other source of thiamine, which is often the case in poor Asian countries. Although the disease has been present in Southeast Asia for thousands of years, it has become more common due to a change in rice processing [9]. It was responsible for over $50 \%$ of deaths among children in the early 20th century in the Philippines [10].

Beriberi is still a big problem in the Karen refugee camps. This is due to a rice-based diet. In addition, they eat a lot of fish paste. It contains the enzyme thiaminase, which breaks down thiamin. Additionally, Karen chew tea leaves and beetles, which results in worse absorption of thiamine from food. Despite the lack of malnutrition in the camps, thiamine deficiency manifests itself in high childhood mortality of 180/1000 live births and in neurological deficits. Currently, thiamine supplementation instead of diet change is the most common practice in the camps [11].

Despite the fact that Malaysia was an endemic place of beriberi, especially before World War II, it is now rarely found in this area [12]. However, it is still seen especially among Burmese refugee camps. The men worked hard physically there, and the meals were low in calories and relied heavily on white rice. In addition, the workers suffered from malnutrition before arriving at the refugee camps [13]. The situation is similar for workers in Vietnam [9] and Thailand [14].

In Africa, the beriberi problem has a different cause than in Asia. It is the sum of alcoholism, hard physical work and a poor, simple diet. This can be seen in the case of the mass incidence of beriberi in the Gambia in 1988. In the town of Chilla, blue-collar workers noticed symptoms typical of beriberi. They started appearing 3 weeks after the begin of the rainy season, when food supplies are the lowest. It is the period of the greatest physical labor in cultivating the land. In addition, it was combined with the heaviest rainfall in 11 years, creating more work. In addition, there is moisture in the stored food. This resulted in an increase in the amount of mycotoxins and thiaminases, which are anti-thiamin factor. 
The diet of manual workers itself was poor in thiamine, based on imported milled rice, and processing during cooking reduced the amount of vitamin B1 in the prepared meal. This resulted in a faster consumption of thiamine resources in working men, which was not supplemented with food [15].

Rarely described, but a common problem is beriberi in prisons. Only in research of The International Comittee of the Red Cross (ICRC) in 2002, checking the health of prisoners in West Africa, noticed symptoms typical of B1 avitaminosis in 211 prisoners. This was the first time beriberi prisoners had been observed in the area, but the disease is suspected to have existed there before. Their diet was mainly based on rice, and access to additional food sources was very limited. Despite the lack of starvation, the lack of thiamine in the diet led to the death of 25 inmates within 11 weeks, and the authorities of the institutions wrongly diagnosed the cause of death as acute renal failure. Similar cases were also reported in prisons in the Caribbean in 1996 and in Rwanda in 1994 [16].

\section{The picture of beriberi disease in highly developed countries}

In highly developed countries, the most important risk factor for thiamine deficiency is alcoholism. There are several mechanisms related to alcohol consumption and vitamin B1 deficiency. One of them is the reduction of thiamine absorption in the small intestine. Another reason is that alcohol consumption reduces caloric intake from sources other than alcohol and thus reduces dietary thiamine intake [3]. Moreover, cirrhosis of the liver, a complication of chronic alcoholism, leads to a reduction of thiamine storage in the liver [5]. Post-mortem studies have shown that the incidence of Wernicke's encephalopathy among alcoholic patients ranges from $12.5 \%$ to $35 \%$, while the incidence of this encephalopathy in the general population is $1.5 \%$ [3].

Refeeding syndrome (RS) is an acute electrolyte deficiency accompanied by fluid retention and impaired glucose metabolism that results from the oral or parenteral nutrition of chronically malnourished patients or those who are re-fed after fasting. The main symptom of $\mathrm{RS}$ is hypophosphatemia, but is often accompanied by other electrolyte abnormalities such as hypomagnesaemia and hypokalaemia. Metabolic disorders lead to numerous complications, including cardiology, neurology, hematology and pulmonology. Patients with proliferative diseases are at particular risk of developing RS. They have both population-specific and individual risk factors. The most dangerous consequences of RS are cardiac complications, which may be caused by thiamine deficiency. RS can develop a "wet" form with peripheral edema and arrhythmias, as well as a "dry" form of beriberi with memory impairment, pathological reflexes, and sensorimotor polyneuropathy. In the case of neurological complications, the risk of Wernicki's encephalopathy or Korsakoff's syndrome results from thiamine deficiency [17].

Gastrointestinal surgery, including surgical treatment of obesity, increases the risk of beriberi. Limiting the size of the stomach reduces the amount of food taken by mouth and therefore a lower intake of vitamins. An additional cause of thiamine deficiency may be postoperative vomiting. 
In patients undergoing gastric bypass surgery Roux-en-Y, another cause of vitamin B1 deficiency is bypass of the duodenum, which is the main site of its absorption, and bacterial overgrowth. It is recommended that all patients should be screened for thiamine deficiency before bariatric surgery and supplemented with vitamin B1 after surgery [1]. Obesity itself is also a risk of vitamin B1 deficiency, due to the fact that these patients use a diet low in vegetables, a simple sugar-rich and processed foods that contain a small amount of vitamins and minerals [3]. In the case of obese people, it should also be remembered that the metabolism of foods containing large amounts of sugar requires more thiamine, and therefore may accelerate its depletion [1].

Patients with heart failure are at risk of thiamine deficiency. This is especially true in patients with congestive heart failure (CHF) taking diuretics. This is due to the increased excretion of thiamine in the urine under the influence of drugs. Moreover, patients with congestive heart failure may be at increased risk of beriberi as a result of disease worsening, malnutrition and advanced age [5].

\section{Treatment}

The therapeutic management of beriberi varies depending on the cause of the thiamine deficiency and clinical symptoms. In patients with alcoholism, the treatment of WernickeKorsakoff syndrome consists of parenteral administration (intramuscularly or intravenously) of 500-1500 mg of thiamine daily in three doses for 5 days. It is important that the initial vitamin intake is parenteral as patients with alcoholism have a significant deficit in thiamine absorption. After this time, it is recommended to administer $300 \mathrm{mg}$ of thiamine orally daily for 1-2 weeks, and then $100 \mathrm{mg}$ daily [3]. Although the daily requirement for thiamine is 1-2 $\mathrm{mg}$, high doses of thiamine in alcohol abusers allow the restoration of vitamin levels, prevent irreversible brain damage, improve clinical condition and prevent death [18]. Upon initiation of vitamin supplementation, ocular symptoms and ataxia should resolve within hours or days, and confusional state disappears within 2-3 weeks. In addition, patients with alcoholism, even without symptoms of vitamin B1 deficiency, should always receive parenteral thiamine prophylactically before starting carbohydrate-containing fluids. This is due to the fact that the supply of glucose increases the need for thiamine and may accelerate the appearance of its deficiency in the patient [19].

Patients diagnosed with Wernicke-Korsakoff syndrome who do not abuse alcohol require lower doses of thiamine than patients with alcoholism. Parenteral treatment in these patients with doses of thiamine 100-200 $\mathrm{mg}$ per day is sufficient to improve the clinical condition.

Treatment of wet beriberi involves oral supplementation. The recommended doses of 100-300 mg daily help to relieve symptoms.

For Shoshin beriberi, thiamine is initially administered parenterally, then orally. Recommended doses are 100-300 mg daily. Vitamin supplementation should be carried out long enough until the cause of thiamine deficiency is removed. Vitamin B1 administration leads to a quick recovery, while if untreated it leads to death [6].

Vitamin B1 supplementation is also indicated in the prevention and treatment of renourishment syndrome. 
The recommended dose of thiamine in patients at risk of RS is 50-100 mg intravenously as a bolus or $100 \mathrm{mg}$ orally per day for the first 3-5 days of nutritional treatment. In the case of large vitamin deficiencies, the supply should start from 200-300 mg per day and continue for 10 days [17].

Oral intake of higher than recommended amounts of thiamine should not cause adverse effects [20]. In contrast, parenteral administration of thiamine can cause serious, but rare complications, such as anaphylaxis and cardiac arrest. In order to reduce the risk of these complications, parenteral thiamine should be diluted in $100 \mathrm{ml}$ of physiological saline and infused over 30 minutes [3].

\section{Summary and conclusions}

The beriberi disease in the world, especially in Asian countries, is mainly due to an inadequate diet, mainly consisting of polished rice and cereals. In African countries, hard physical labor is another major cause of thiamine deficiency. In Western countries, it most often occurs in patients who abuse alcohol or suffer from chronic disease. The apparently easy to treat beriberi disease can cause many diagnostic difficulties. The symptoms of thiamine deficiency are nonspecific, affect many organs, and can lead to severe and potentially irreversible neurological damage and even death. The overall prognosis for patients with beriberi is good because it is easily treated. Most symptoms of deficiency resolve when thiamine supplementation is introduced. However, When a vitamin B1 deficiency develops into Wernicke-Korsakoff syndrome, initial treatment may improve, but symptoms may be permanent. Prevention of beriberi is easy, that is, it consists in ensuring the appropriate level of thiamine necessary for participation in metabolic processes. It is imperative to educate patients at risk of thiamine deficiency, first of all about the causes of its deficiency, a healthy diet and abstaining from alcohol. Thanks to this approach, it will be possible to reduce the morbidity associated with vitamin B1 deficiency. It is imperative to educate patients at risk of thiamine deficiency, first of all about the causes of its deficiency, a healthy diet and abstaining from alcohol. Thanks to this approach, it will be possible to reduce the morbidity associated with vitamin B1 deficiency. It is imperative to educate patients at risk of thiamine deficiency, first of all about the causes of its deficiency, a healthy diet and abstaining from alcohol. Thanks to this approach, it will be possible to reduce the morbidity associated with vitamin B1 deficiency. 


\section{List of references}

1. Kerns J, Arundel C., Chawla L. Thiamin Deficiency in People with Obesity. Advances in Nutrition. 2015; 6 (2): 147-153.

2. Lonsdale D. Thiamin. New Research and Developments of Water-Soluble Vitamins. 2018. 1-56.

3. Polegato B., Pereira A., Azevedo P., et al. Role of Thiamin in Health and Disease. Nutrition in Clinical Practice. 2019. 1-7.

4. Wiley KD. Vitamin B1 Thiamine Deficiency (Beriberi). In: StatPearls Publishing [Internet]. 2020. https://www.ncbi.nlm.nih.gov/books/NBK537204/

5. Frank LL. Thiamin in Clinical Practice. Journal of Parenteral and Enteral Nutrition. 2015; 39 (5): $503-$ 520.

6. Dabar, G., Harmouche, C., Habr, B., Riachi, M., \& Jaber, B. Shoshin Beriberi in Critically-Ill patients: case series. Nutrition Journal. 2015; 14 (1).

7. Gerridzen IJ, Joling KJ, Depla MF, et al. Awareness and its relationships with neuropsychiatric symptoms in people with Korsakoff syndrome or other alcohol-related cognitive disorders living in specialized nursing homes. Int J Geriatr Psychiatry. 2019; 34 (6): 836-845.

8. Pietrzak K. Christiaan Eijkmann (1856-1930). Journal of Neurology. 2019; 266: 2893 - 2895.

9. Nguyen PAH, Pittayawonganon C, Praekunatham H, et al. Beriberi outbreak among factory workers in Chachoengsao Province, Thailand, 2012-2013. OSIR. 2014; 7 (3): 1-7.

10. Gregg D. Infantile Beriberi in the Philippines. Boston Med Surg J. 1913; 168: 676-8.

11. Nosten F., Beriberi in Cambodia. Paediatrics and International Child Health. 2015; 35 (4): $283-284$.

12. Jeyakumar D. Thiamine responsive ankle oedema in detention center inamtes. Med J Malays. $1995 ; 50$ (1): 17-20.

13. Sunita AR., Mohannad SJ., Fadzilah K., et al. Beriberi Outbreak Among Unauthorised Immigrants in a Detention Camp in Malaysia. Journal of Immigrant and Minority Health. 2018; 20 (5): 1294-1297.

14. Doung-ngern P., Kesornsukhon S., Kanlayanaphotporn J., Wanadurongwan S., Songchitsombon S. Beriberi outbreak among commercial fishermen, Thailand 2005. Southeast Asian J Trop Med Public Health. 2007; 38 (1): 59-64.

15. Tang CM, Rolfe M., Wells JC, Cham K., Outbreak of beriberi in the Gambia, The Lancet 1989; 2 (8656): 206-7.

16. de Montmollin D., MacPhail J., McMahon J., Coninx R., Outbreak of beriberi in a prison in West Africa. Tropical Doctor. 2002; 32: 234-236.

17. Püsküllüoglu M, Nieckula J, Laprus I. Re-nourishment syndrome in cancer patients. [Refeeding syndrome in oncological patients]. Via Medica. 2011; 7 (1): 24-30. Polish.

18. Latt, N., \& Dore, G. Thiamine in the treatment of Wernicke encephalopathy in patients with alcohol use disorders. Internal Medicine Journal. 2014; 44 (9): 911-915.

19. Soyka, M., Kranzler, HR., Hesselbrock, et al. Guidelines for biological treatment of substance use and related disorders, part 1: Alcoholism, first revision. The World Journal of Biological Psychiatry. $2016 ; 18$ (2): 86-119.

20. Brown G. Defects of thiamine transport and metabolism. Journal of Inherited Metabolic Disease. 2014; 37 (4): 577-585. 